\title{
Cryogenic Atomic Resolution and 4D STEM Imaging for Energy and Quantum Materials
}

\author{
Miaofang Chi ${ }^{1}$ and Andrew R. Lupini ${ }^{2}$ \\ ${ }^{1}$ Center for Nanophase Materials Sciences, Oak Ridge National Laboratory, Oak Ridge, Tennessee 37831, \\ USA, United States, ${ }^{2}$ Oak Ridge National Laboratory, United States
}

Four-dimensional STEM (4D-STEM) has been attracting significant attention in recent years in the electron microscopy community. In this method, nearly all electrons are collected after they interact with specimen. A 'complete' set of information about the specimen is encoded in 4D-STEM datasets and can be reconstructed via applying various phase retrieval methods or virtual detectors. In particular, information such as highresolution charge density mapping, local potential distributions and chemical bonding, which were challenging to obtain before, have recently been demonstrated [1-6]. These new capabilities are critical to the research of quantum and energy materials, where local charge and potential define the function of materials and devices. However, many of the materials in these two categories require the characterization to be performed at a low temperature. For example, exotic states in quantum materials usually only occur at a low temperature (T); and lithium metal and solid electrolyte interphase layers in battery materials are extremely sensitive to electron irradiation induced heating. The largest challenge in in situ cryo-STEM for materials science currently is the limited stage and temperature stability, making imaging at atomic resolution or at intermediate temperatures difficult [7]. Achieving a sufficient stability is even more challenging for Cryo-4D-STEM, where a longer acquisition is usually needed than for STEM imaging using a conventional detector.

In this work, we focus on configuring atomic resolution and Lorentz mode 4D-STEM imaging at cryo-T on a JEOL-NeoARM microscope at ORNL. The applications of these techniques are demonstrated by using several model quantum materials. First, we will assess the stability of several commercial liquid nitrogen holders from different manufactories. The temperature and tilting range each holder extents, the stability for atomic resolution imaging at baseline and intermediate temperatures will be evaluated. Figure 1 shows the performance of atomic-resolution STEM imaging at $120 \mathrm{~K}$ with one of the cooling holders at ORNL. An example image of $512 \times 512$ pixels with a dwell time of $4 \mu \mathrm{s} /$ pixel is shown in Figure 1a. The signal-to-noise ratio of the images is significantly improved by summing-up multiple fast scans from the same specimen region as shown in Figure 1b. Specimen drifts (Fig. 1c) in random directions but stayed within $1 \AA$ range in 120 s. These images demonstrate both the feasibility and difficulty of atomic resolution imaging at intermediate temperatures. The second topic of this presentation will report our progress on configuring cryo-Lorentz 4DSTEM imaging. Setting up Lorentz 4D-STEM on a microscope is not new [8-10]. However, the achievable spatial resolution of such configuration, especially with the integration of a cryo-stage, merits investigation. Compared to cryo-STEM imaging, cryo- Lorentz 4D-STEM imaging at a high spatial resolution faces additional challenges. Not only is a longer acquisition time needed, but the Lorentz mode lens configuration usually impairs the electron probe stability. This is because, unless a special lens design is used [5], the objective lens is either turned off or operated at a weak strength, resulting in a larger focal length than in the regular STEM imaging mode. The probe stability can be significantly affected by both external fields and intrinsic lens stability. Our experiments demonstrate that a $\sim 4 \mathrm{~nm}$ spatial resolution of Lorentz STEM on the NeoARM is achievable, which is adequate to image magnetic domains in most magnetic materials. Figure 2 shows an example of imaging a skyrmion specimen using cryo- Lorentz 4D-STEM. An acquisition of 128x128 points at 264x264 pixels with a camera speed of $1 \mathrm{kfps}$ was used. Magnetic fields of features as small as $8 \mathrm{~nm}$ are clearly revealed. Strategies and challenges in further improving the spatial resolution of Lorentz STEM and the feasibility of performing cryo- Lorentz STEM at intermediate temperatures will also be discussed [11]. 

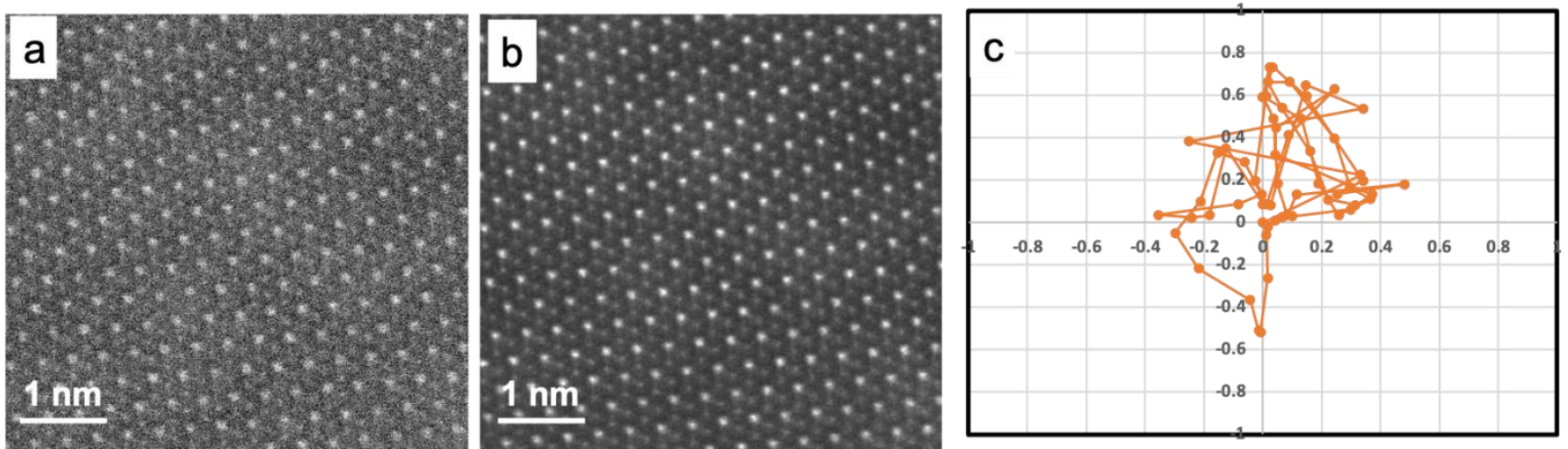

Figure 1. Figure 1. HAADF-STEM images of BaFe2A19 at $120 \mathrm{~K}$ showing (a) a single-scan with a pixel time of 4 microseconds, (b) a summed image of ten sequential scans after being aligned with cross-correlation registration, (c) the drift of the specimen in both $\mathrm{x}$ and y directions in $\AA$ with a total recording time of 120 s.
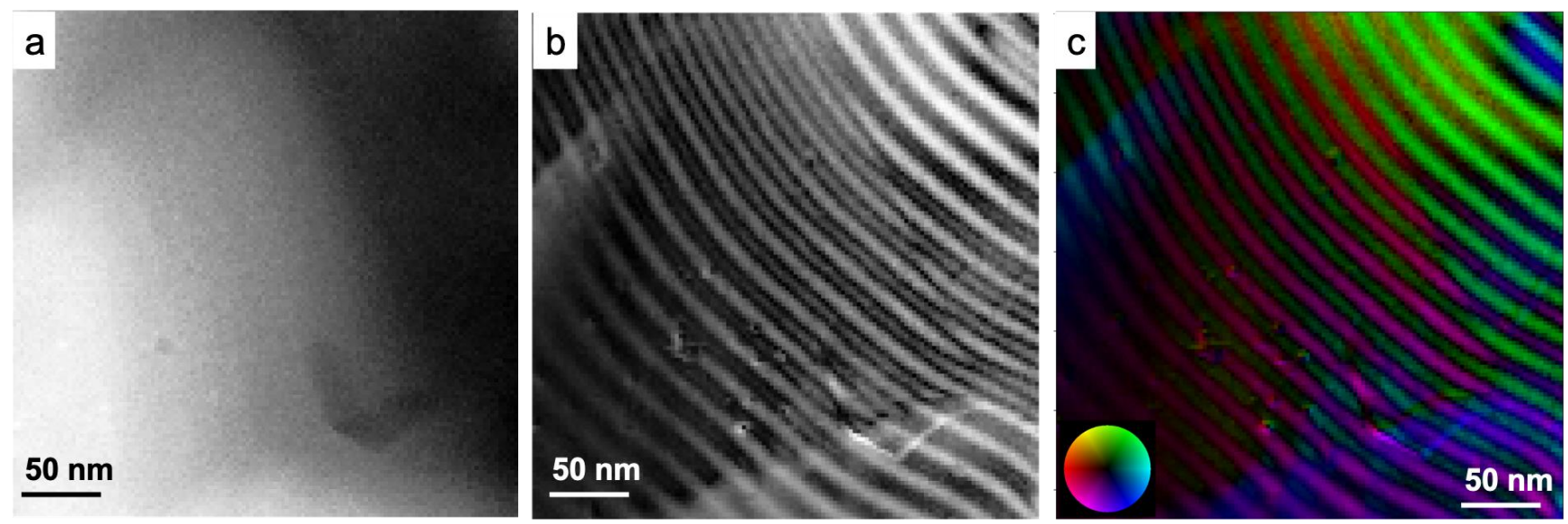

Figure 2. Figure 2. Reconstructed images of a typical region in skyrmion FeGe1-xSix from a Lorentz 4DSTEM dataset acquired at 108K, including a HAADF image (a); and an electric field map reconstructed by tracing the diffraction central disk (b); and a color vector map of the in-plane magnetization (c).

\section{References}

1. Ophus, C. 25, 563-582 (2019).

2. Hachtel, J. A., Idrobo, J. C. \& Chi, M. 4, 10 (2018).

3. Qiang, Z. et. al., Science Adv. (2021).

4. Jiang, Y. et al. Nature 559, 343-349 (2018).

5. Shibata, N. et al. Nat. Commun. 10, 1-5 (2019).

6. Martinez, G. T. et al. arXiv (2019).

7. Goodge, B. H. et al. 26, 439-446 (2020).

8. Nguyen, K. X. et al. Microsc. Microanal. Microsc. Microanal. 21, S3 (2015).

9. Zaluzec, N. J. Microsc. Microanal. 7, 222-223 (2001).

10. McVitie, S. et al. Sci. Rep. 8, 5703 (2018).

11. This work was supported by DOE Office of Science Basic Energy Sciences Early Career project ERKCZ55 (MC) and, Division of Materials Science and Engineering (AL). Experiments were performed at the Center for Nanophase Materials Sciences (CNMS), which is a DOE Office of Science User Facility. 